\title{
Economic evaluation of End Stage Renal Disease treatments in Iran
}

\author{
Amirali Moradpour ${ }^{\mathrm{a}}$, Mohamad Hadian ${ }^{\mathrm{b}, *}$, Mahmoud Tavakkoli $^{\mathrm{c}}$ \\ ${ }^{a}$ Department of Health Economics, School of Health Management and Information Science, Iran University of Medical Sciences, Tehran, Iran \\ ${ }^{\mathrm{b}}$ Health Management and Economics Research Center, Iran University of Medical Sciences, Tehran, Iran \\ ${ }^{\mathrm{c}}$ Kidney Transplantation Complications Research Center, Mashhad University of Medical Sciences, Mashhad, Iran
}

\section{A R T I C L E I N F O}

\section{Keywords:}

Iran

Cost-utility analysis

Hemodialysis

Peritoneal dialysis

Kidney transplantation

\begin{abstract}
A B S T R A C T
Background: End-stage Renal Disease is considered a health problem due to the high prevalence and economic burden on society and the health system. This study utilizes a cost-utility analysis to evaluate the costs and outcomes of the Iranian End Stage Renal Disease patients.

Methods: A Markov model-based economic evaluation with a societal perspective, and a lifetime horizon performed to quantify the costs and health-related outcomes in terms of QALY. Direct medical costs obtained from hospital billing and medical records and direct non-medical costs, and indirect costs derived from interviews with patients. Three policy options, the hemodialysis, peritoneal dialysis and kidney transplantation were compared. Most of the Transplants from deceased and rest were from Live Related Donors. One-way and probabilistic sensitivity analyses were performed to study uncertainty.

Results: Annual average cost of hemodialysis is $\$ 13477$ cost of peritoneal dialysis is $\$ 12865$, and cost of Transplantation is \$16450.The Transplantation arm gained 9.43 QALY compared with peritoneal dialysis and hemodialysis with 6.95 and 6.04 QALY respectively. When Transplantation was compared with peritoneal dialysis, Incremental Cost-Effectiveness Ratio was \$1744 per QALY. ICER value suggests that Transplantation is cost-effective compared with peritoneal dialysis at a willingness-to-pay threshold of $\$ 12,400$, and hemodialysis was dominated.

Conclusion: This study suggests that kidney transplantation is a better option over hemodialysis and peritoneal dialysis. We conclude that serious efforts ought to be made to foster potential brain-dead donors and altruistic kidney donation and promote peritoneal dialysis as a superior alternative to hemodialysis for eligible patients.
\end{abstract}

\section{Introduction}

Patients in the End Stage Renal Disease (ESRD) are being treated with one of the Renal Replacement Therapy (RRT) methods including hemodialysis (HD), peritoneal dialysis (PD), and kidney transplantation (TX). ${ }^{1}$ Despite the advances in diagnosis and treatment, the morbidity and mortality rates of ESRD are high. ${ }^{2}$ World's population increased $1.1 \%$ annually, while the number of patients with ESRD increased by approximately $6 \% .^{3}$

In parallel with the advances in medicine, kidney transplantation is one of the most preferred and outstanding treatment methods for ESRD organ failure, which provides significant benefits in terms of medical, social and quality of life, costs and reduction in labor loss. But not all patients can undergo transplantation, and treatment availability is low for a shortage in donor kidneys. Not all patients are able to undergo transplantation. ${ }^{4}$ Estimates on the Global Burden of Disease (GBD) indicated that kidney diseases were responsible for 2,993,000 years of life lost (YLL) and 38,104,000 disability adjusted life years (DALYs) lost globally. ${ }^{5}$

ESRD is one of the most resource-consuming diseases and the increasing frequency of the disease causes more financial resources than the countries' budget. The high cost of renal replacement therapy (RRT) presents an economic and ethical dilemma for those responsible for funding treatment programs for patients with end-stage renal disease (ESRD). ${ }^{6}$

According to the 2017 Iran consortium of Dialysis (ICD) report, the prevalence of ESRD in Iran was 680 per million. ${ }^{7}$ The main aim of RRT treatment is to prolong the lifetime of the patients and increase the quality of their life. ${ }^{8}$ By the end of 2017 in Iran, 58,000 patients treated with one of these methods. Hemodialysis was the primary treatment of the patient in Iran with almost $50 \%$ followed by transplantation and peritoneal dialysis with47 and $3 \% .^{7}$

As for the total cost of the RRT programs in Iran, most of the estimated costs were attributable to $\mathrm{HD}$ and $\mathrm{TX}$, and only small percentages

\footnotetext{
* Corresponding author.

E-mail addresses: moradpour.a@iums.ac.ir (A. Moradpour), hadian.m@iums.ac.ir (M. Hadian), tavakkolim@mums.ac.ir (M. Tavakkoli).
} 
Table 1

Baseline characteristics of patients with ESRD.

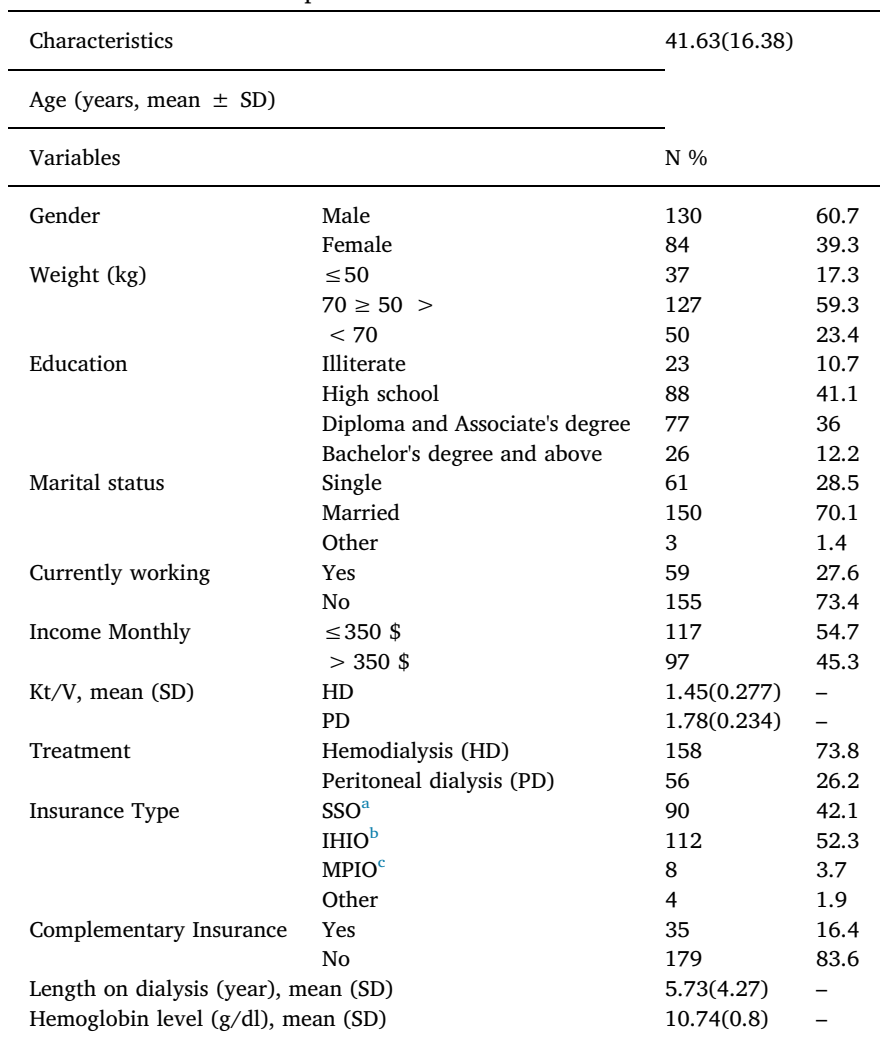

a Social Security Organization (SSO).

b Iran Health Insurance Organization.

c The Military Personnel Insurance Organization (MPIO).

were because of PD. In the present study, we aimed to determine which treatment strategies should implement to cover all patients and to assess the costs and health outcomes of the Iranian ESRD patients.
Table 2

The incremental cost-effectiveness ratio (ICER) of options.

\begin{tabular}{lllllll}
\hline Strategy & Cost \$ & Incr Cost & Eff & Incr Eff & ICER & Average CE \\
\hline $\begin{array}{l}\text { Peritoneal dialysis } \\
\begin{array}{c}\text { Hemodialysis } \\
\quad \text { (Dominated) }\end{array}\end{array}$ & 12865 & 0 & 6.951 & 0 & 0 & 1850 \\
\begin{tabular}{c} 
Transplantation \\
\hline
\end{tabular} & 16449 & 612 & 6.049 & -0.901 & -679 & 2227 \\
\hline
\end{tabular}

\section{Methods}

Research Method and patients descriptions : To compare the cost-effectiveness of RRT treatment methods, a cost-utility analysis performed based on the societal perspective and lifetime horizon. Total of 214 patients recruited from two hospitals (Imam Reza and Montaserieh) in Mashhad, Iran with 56 patients for PD and 158 for HD. The inclusion criteria for the study population were ESRD patients treated with $\mathrm{HD}$ or $\mathrm{PD}$, adults (older than 18 years old), had received a minimum of a three-month therapy with either HD or PD, and had no contraindications of both modalities (including the incapability of doing PD at home). Patients who discontinued dialysis therapy within three months or had combination therapy of both HD and PD excluded from the study. Most of the Transplants from deceased and rest were from Live Related Donors. Table 1 gives a description of our study population.

Model: Four-State Markov model with TreeAge version 2011 software used to measure the cost-effectiveness of ESRD. The model states include HD, PD, TX, and Dead which were determining the states of ESRD.

According to expert and previous studies ${ }^{4} 9-11$ these states can change in such way that HD patients can maintain the same state or go to other three states. Also this is the case for PD and TX patient's states. The "Dead" state is absorbed state (Fig. 1). The model used a one-month cycle length for health state. Lifetime horizon applied in this model. Using this approach, total lifetime cost and health outcomes amongst the four policy options compared.

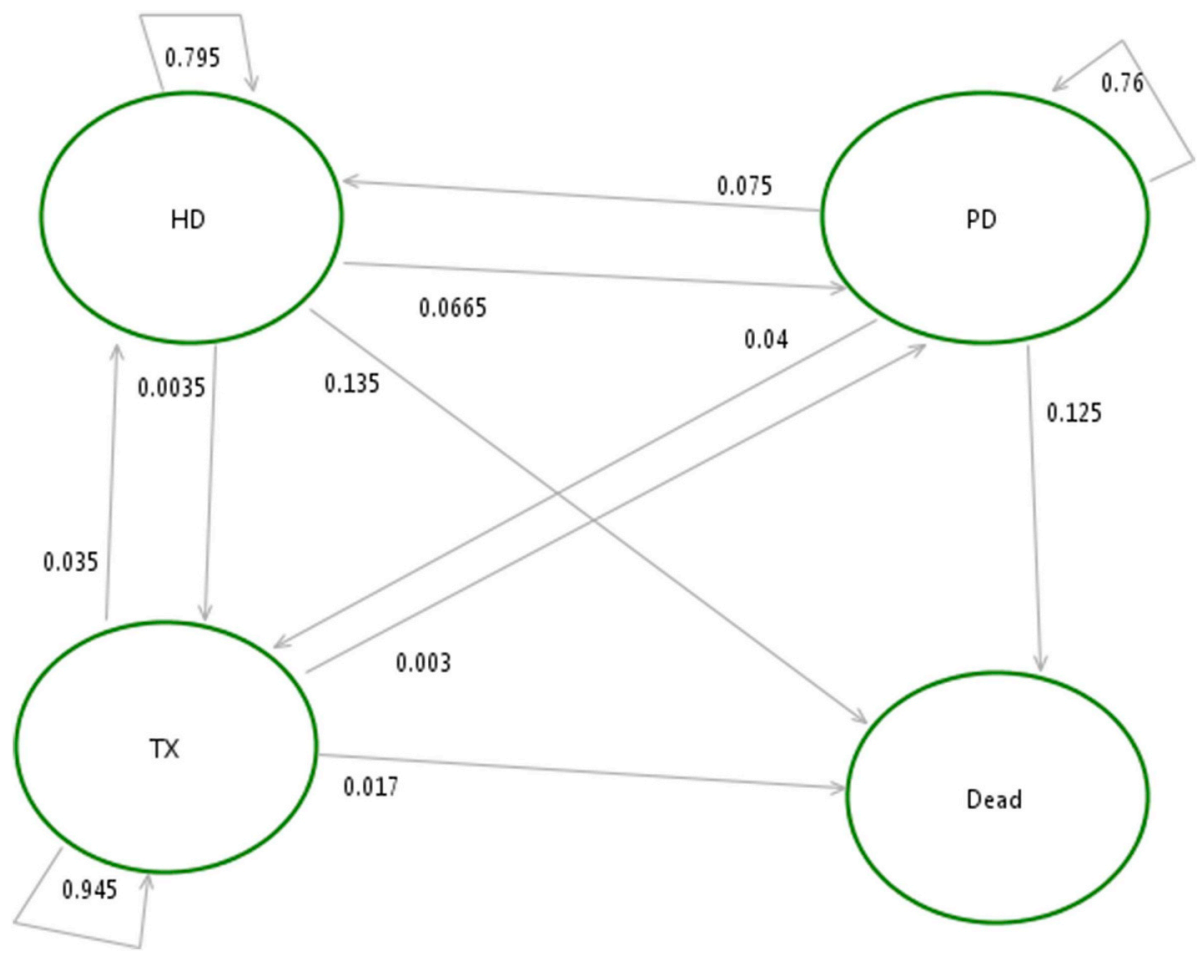

Fig. 1. Schematic diagram of Markov model. 


\section{CE Acceptability Curve}

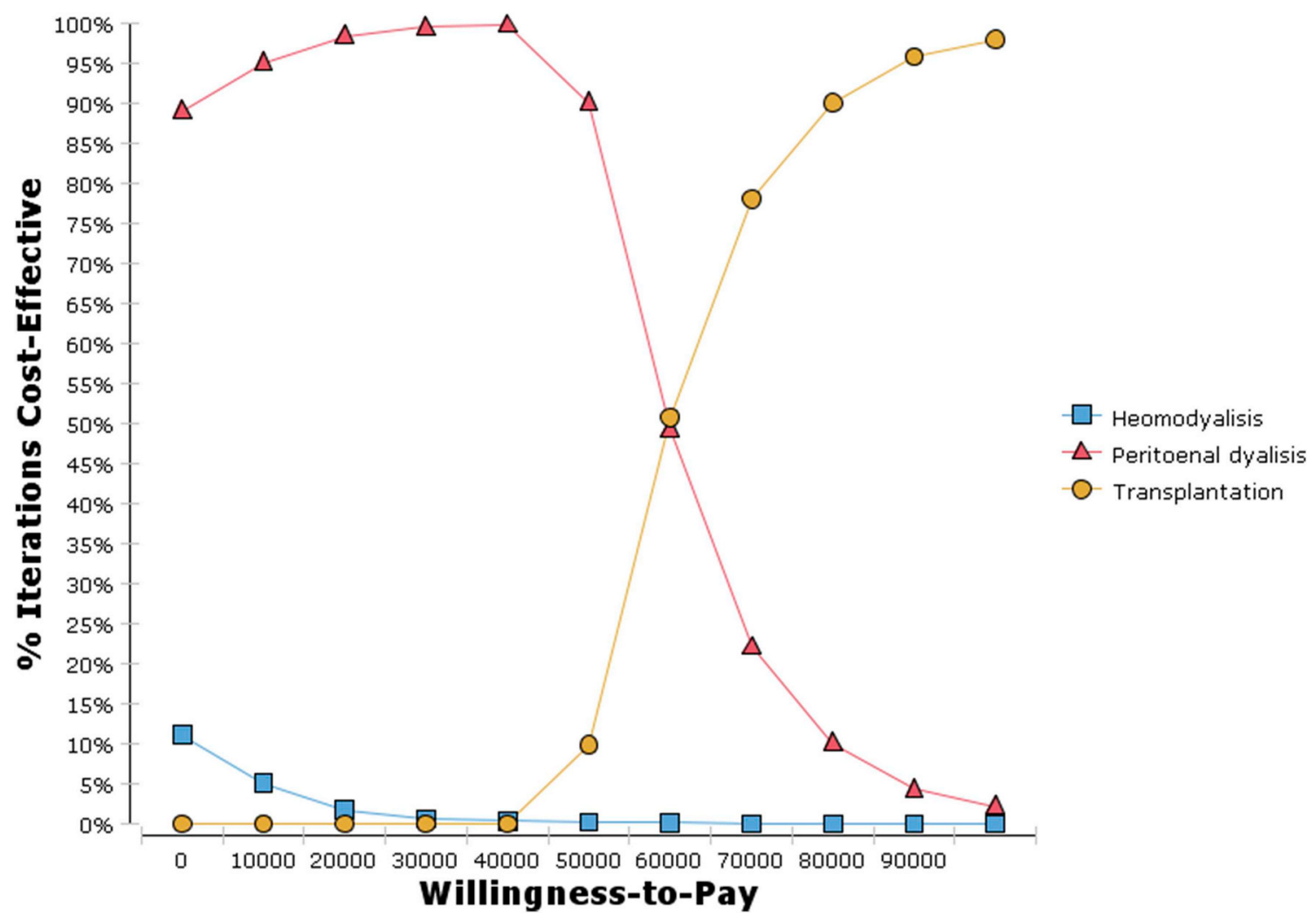

Fig. 2. The cost-effectiveness acceptability curve.

Cost and outcome: Based on societal perspectives direct medical, direct non-medical and indirect costs included in the analysis. The data obtained primarily from hospital billing and medical records for a oneyear period. Direct non-medical costs, and indirect costs derived from interviews with patients using a questionnaire. For direct non-medical costs, all subjects asked about their travel, food, and accommodation expenses when they visited hospitals. The direct non-medical costs pertained to the costs consumed by patients and caregivers while the indirect costs referred to the income loss of the caregiver in a year.

All costs presented in the year 2017 and discounted at a rate of $6.0 \%$ based on local discount rates ${ }^{12,13}$ for both costs and outcomes. The country's threshold is three times of the country's GDP per capita (about 520 million IRR ( $\$ 12380$ ) per QALY) applied in this study. All costs calculated based on the value of US dollar in $2017 .{ }^{14}$ Utility values obtained from the Euro QoL EQ-5D-5L Persian version. ${ }^{15}$ Patients asked about their current condition and their condition during states. The utility data of $\mathrm{HD}, \mathrm{PD}$, and TX patients, was 0.72 and 0.75 , and 0.82 respectively.

\section{Sensitivity analysis}

Considering uncertain parameters of the model including QALY, initial distribution and the transition probabilities, basic and probabilistic sensitivity analysis carried out using variation range of $20 \%$, and calculating the costs using the lowest and highest amounts.

A probabilistic sensitivity analysis using a Monte Carlo simulation conducted in this study. All input parameters assigned a probability distribution to reflect a possible range of its values. The process repeated for 1000 simulations. Each simulation provided one value of cost-effectiveness. The average value of the probabilistic sensitivity analysis presented as the ICER value and the cost-effectiveness acceptability curve.

Results

Costs: Using the societal perspective, the total annual costs of HD was 566, 055, 000 IRR ( $\$ 13477$ ) cost of PD 540,331,000 IRR (\$12865 ), and cost of TX was $690,882,000$ IRR ( $\$ 16450$ ).

The HD had the highest costs among the three options and showed a large burden borne by households whereas the PD was the least costly options. The TX arm gained 9.43 QALY compared with PD and HD with 6.95 and 6.04 QALY respectively.

Base case analysis: The incremental cost-effectiveness ratio (ICER) of providing the TX was $\$ 1744$ per QALY gained, which was lower than the PD with $\$ 1850$, and HD with $\$ 2227$ per QALY gained (Table 2 ). The cost-effectiveness acceptability curve (Fig. 2) showed the probability of favoring each option dependent on the level of willingness to pay. At willingness to pay $\$ 12380$, TX was the best option.

Sensitivity Analysis: For all uncertain parameters of the model including QALY, the cost, transition probability, and the initial distribution based on variation range, the basic sensitivity analysis performed. According to the tornado diagram (Fig. 3), three first parameters had the greatest impact on costs. One-way sensitivity analyses performed on the most-influential variables in the model. The utility of TX, discount rate for outcomes, utility of HD, and cost of TX noted to be the most sensitive in the mode. According to the results of the analysis result was not sensitive to changes of these variables.

A probabilistic sensitivity analysis performed using a Monte Carlo simulation of 1000 trials and showed that the probability of TX being more cost-effective at a willingness-to-pay threshold of $\$ 12380$ (about 520 million IRR) was $54.5 \%$. Fig. 4 depicts these data in the form of an ICER scatter plot, with the drawn circle representing the $95 \%$ CI. 


\section{Tornado Analysis (Net Benefits)}

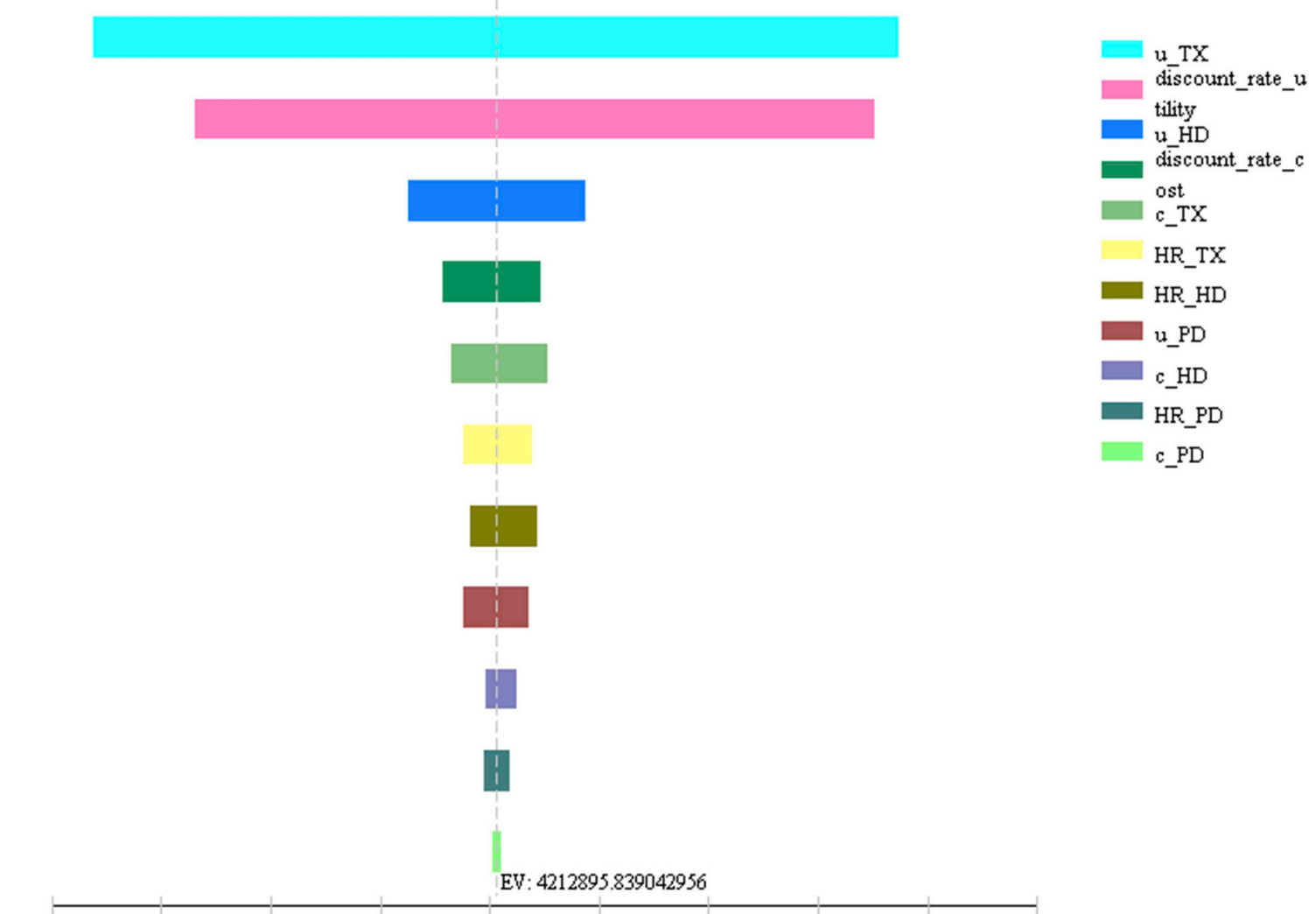

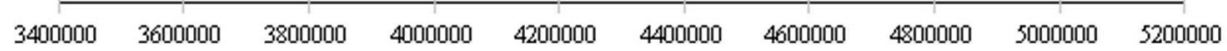

Fig. 3. The Tornado diagram.

\section{Incremental Cost-Effectiveness, Transplantation v. Peritoenal dyalisis}

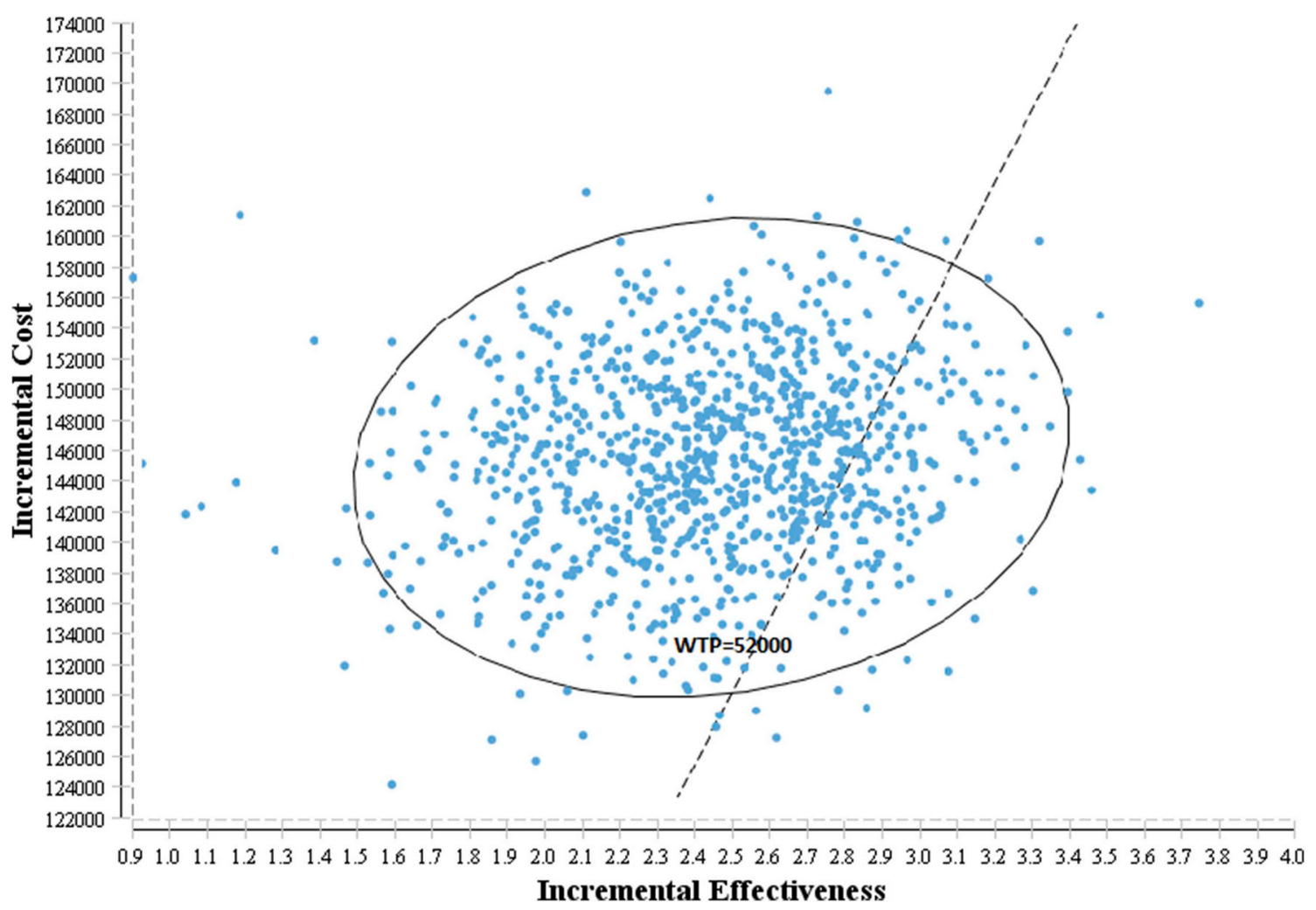

Fig. 4. ICER scatter plot. 


\section{Discussion}

Our study showed that kidney transplantation and peritoneal dialysis perform better when compared to hemodialysis and Kidney Transplantation is more cost-effective, representing a savings of \$1477 per patient/y.

The mean annual cost of HD, PD, and TX of Iran was about $\$ 13477$, 12865 , and 16450 respectively that in compare to Turkey ( $\$ 14652$, 14389, and 25390), ${ }^{16}$ Taiwan (\$16643 and 13681, NA), ${ }^{17}$ Canada ( $\$ 43816$ and 38658, NA), ${ }^{18}$ and USA (\$72486, NA, and 80486), ${ }^{19}$ so Iranian ESRD costs had an intermediate level. The HD had the highest costs among the three options and showed a large burden borne by households whereas the PD was the least costly options.

The fact that renal transplantation yields more favorable CostEffectiveness than dialysis therapies has been previously demonstrated in several studies. Our findings are consistent with Howard et al. that explained clinical practice changes reduce costs, improve patient quality of life and, in the case of transplantation, increase survival. ${ }^{20}$ Haller et al. described that live donor renal transplantation is cost effective and associated with increase in QALYs. ${ }^{4}$ Yigit et al. found that kidney transplantation treatment had better cost saving compared to dialysis and the cost per QALY was lower, and the quality of life and survival rate of kidney transplantation patients were higher than HD and PD. ${ }^{16}$ Some other studies regard that hospital hemodialysis being the least cost-effective treatment option for patients with terminal kidney failure. ${ }^{20-22}$ Peritoneal dialysis and kidney transplantation represent more cost-effective therapy strategies. ${ }^{21,} 23-25$

According to the results of the study, Kidney transplantation treatment should be preferred and encouraged when developing health policies and allocating resources to health care services because of costeffectiveness in terms of transplantation, quality of life, patient survival and cost of treatment. In face of these findings serious efforts ought to make to foster not only altruistic living kidney donation but also more effective recruitment of potential brain-dead donors on the one hand and promote peritoneal dialysis as a superior alternative to hemodialysis for eligible patients on the other.

In summary, in our study, we found that Kidney transplantation was somewhat more cost-effective than Hemodialysis and Peritoneal dialysis but caution has to be exercised when the results are interpreted. Choosing between the modalities is a complex decision, where both medical and non-medical factors attribute selection. In addition serious efforts ought to be made to foster potential brain-dead donors and altruistic kidney donation and promote peritoneal dialysis as a superior alternative to hemodialysis for eligible patients.

Our study has some limitations. Firstly, we used the calculated QALY value of other countries because this value has not been calculated in our country yet, so we used sensitivity analysis for determining the robustness of the results. Secondly, the data were drawn from a small group of patients with ESRD and we did not include all the patients from the country. Finally, our study has the lack of follow up period, our patients investigated for one year and we need to follow up patients.

\section{Ethical issues}

Informed consent forms were obtained from all subjects participated in the study. The Ethics Committee of Iran University of Medical Sciences concurred with the study protocol (IR.IUMS.REC.1395. 9221504205).

\section{Conflict of interest}

The authors declared no conflict of interest regarding the used materials and the reported results.

\section{Authors' contributions}

Amirali Moradpour: Conception of the study, drafting the article, final approval of the version to be published. Mohamad Hadian: Conception of the study, revising the article, final approval of the version to be published. Mahmoud Tavakkoli: Analysis and interpretation of data, revising the article, providing intellectual content of critical importance to the work described; and final approval of the version to be published.

\section{Funding}

This study was part of a PhD thesis supported by the Iran University of Medical Sciences (IUMS/SHMIS- 1395- 9323504001).

\section{Acknowledgments}

This study was part of a PhD thesis supported by the Iran University of Medical Sciences (IUMS/SHMIS- 1395- 9323504001).

\section{Appendix A. Supplementary data}

Supplementary data to this article can be found online at https:// doi.org/10.1016/j.cegh.2019.07.001.

\section{References}

1. Just PM, Riella MC, Tschosik EA, Noe LL, Bhattacharyya SK, de Charro F. Economic evaluations of dialysis treatment modalities. Health Policy. 2008;86:163-180.

2. Burrows NR, Hora I, Geiss LS, Gregg EW, Albright A. Incidence of end-stage renal disease attributed to diabetes among persons with diagnosed diabetes - United States and Puerto Rico, 2000-2014. MMWR Morb Mortal Wkly Rep. 2017;66:1165-1170.

3. Fresenius Medical Care. EPiAGP. Fresenius Medical Care. (2017). ESRD Patients in 2012: A Global Perspective., 2017. 2017; 2017.

4. Haller MG, Kramar G, Harnoncourt R, Oberbauer F. Cost-effectiveness analysis of renal replacement therapy in Austria. Nephrol Dial Transplant. 2011;26:2988-2995.

5. World Health Organization. Global Burden of Disease.: Regional Estimates for 2000-2016. DALY Estimates.; 2017.

6. Salonen T, Reina T, Oksa H, Rissanen P, Pasternack A. Alternative strategies to evaluate the cost-effectiveness of peritoneal dialysis and hemodialysis. Int Urol Nephrol. 2007;39:289-298.

7. Iranesrd.com. 2016; 2016www.iranesrd.comPublications.html.pdf.

8. Afiatin Khoe LC, Kristin E, et al. Economic evaluation of policy options for dialysis in end-stage renal disease patients under the universal health coverage in Indonesia. PLoS One. 2017:12:e0177436.

9. Yiğit V. Economic Evaluation of Health Care: Cost-Effectiveness Analysis of the Treatment of Dialysis and Kidney Transplantation in Turkey [dissertation]. SDU Institute of Social Sciences; 2013

10. Villa G, Fernandez-Ortiz L, Cuervo J, et al. Cost-effectiveness analysis of the Spanish renal replacement therapy program. Perit Dial Int. 2012;32:192-199.

11. Rosselli Diego J-DR, Eduardo Diaz Carlos. Cost-effectiveness of kidney transplantation compared with chronic dialysis in end-stage renal Disease.pdf. Saudi J Kidney Dis Transpl. 2015;26(4) 733-738 2015.

12. Daneshmand A, Jahangard E, Abdollah-Milani M. A time preference measure of the social discount rate for Iran. J Econ Struct. 2018;7:29.

13. Mahboub-Ahari A, Pourreza A, Sari AA, Rahimi Foroushani A, Heydari H. Stated time preferences for health: a systematic review and meta analysis of private and social discount rates. J Res Health Sci. 2014;14:181-186.

14. Central bank of Islamic Republic of Iran er. Iran, Central Bank of Islamic Republic of Iran. Exchange Rate. 2017; 201720172017.

15. Ameri H, Yousefi M, Yaseri M, Nahvijou A, Arab M, Akbari Sari A. Mapping the cancer-specific QLQ-C30 onto the generic EQ-5D-5L and SF-6D in colorectal cancer patients. Expert Rev Pharmacoecon Outcomes Res. 2019;19:89-96.

16. yiğit V. Cost-effectiveness analysis of dialysis and kidney transplantation treatment in Turkey. Bilig Turk Dunyasi Sosyal Bilimler Dergisi. 2015;7:82-205.

17. Chang Y-T, Hwang J-S, Hung S-Y, et al. Cost-effectiveness of hemodialysis and peritoneal dialysis: a national cohort study with 14 years follow-up and matched for comorbidities and propensity score. Sci Rep. 2016;6:30266.

18. Beaudry A, Ferguson TW, Rigatto C, Tangri N, Dumanski S, Komenda P. Cost of dialysis therapy by modality in manitoba. Clin J Am Soc Nephrol. 2018;13(8):1197-1203. https://doi.org/10.2215/CJN.10180917.

19. Axelrod DA, Schnitzler MA, Xiao H, et al. An economic assessment of contemporary kidney transplant practice. Am J Transplant. 2018:18:1168-1176.

20. Howard K, Salkeld G, White S, et al. The cost-effectiveness of increasing kidney transplantation and home-based dialysis. Nephrology. 2009;14:123-132.

21. De Wit GA, Ramsteijn PG, de Charro FT. Economic evaluation of end stage renal disease treatment. Health Policy. 1998;44:215-232. 
22. Jarl J, Gerdtham U-G. Economic Evaluations of Organ Transplantations - A Systematic Literature Review. vol. 1. 2011; 20112011.

23. SalonenTapani. Economic Evaluation of Renal Replacement Therapies [PhD dissertation]. University Of Tampere; 2016.
24. Sennfalt K, Magnusson M, Carlsson P. Comparison of hemodialysis and peritoneal dialysis-a cost-utility analysis. Perit Dial Int. 2002;22:39-47.

25. Laupacis A, Muirhead N, Keown P, Wong C. A disease-specific questionnaire for assessing quality of life in patients on hemodialysis. Nephron. 1992;60:302-306. 